\title{
Malignant Peripheral Nerve Sheath Tumour of the Breast in Complex Clinical Background
}

\author{
Arnis Abolins*/*, Andrejs Vanags*/*, Guna Volanska*/*, Inga Melbarde-Gorkusa*/*, Ilze Strumfa*, Genadijs \\ Trofimovics*/**, Janis Gardovskis*/** \\ * Hereditary Cancer Institute, Riga Stradins University, Riga, Latvia \\ ** Pauls Stradins Clinical University Hospital, Riga, Latvia
}

\section{Summary}

Here we describe a patient presenting with combination of three rare diseases, namely de novo malignant peripheral nerve sheath tumour of the breast, small cell variant of medullary thyroid carcinoma and multiple sclerosis. Although the combination by chance seems unlikely, the detailed history does not correspond to any of the known hereditary cancer syndromes and hypothetically may represent a new entity.

Key words: malignant peripheral nerve sheath, tumour, breast.

\section{AIM OF THE DEMONSTRATION}

Breast cancer is the most common cancer among women in European Union (5). The vast majority of breast malignancies are carcinomas, but non-epithelial malignancies may also involve the breast.

The aim of the case report is to demonstrate very rare oncological situation involving de novo malignant peripheral nerve sheath tumour (MPNST) of the breast associated with medullary thyroid cancer in the setting of multiple sclerosis.

\section{CASE REPORT}

A 46-year-old female, suffering from multiple sclerosis, was consulted in the Pauls Stradins Clinical University Hospital because of large breast tumour that has increased slowly during 5 years. The family history was unremarkable. By objective examination, firm tumour, measuring $15 \mathrm{~cm}$, was palpable in the centre of the breast and $6 \mathrm{~cm}$ large, firm mass was palpable in right axilla. During extensive clinical evaluation, core biopsy was performed. It revealed solid, anaplastic tumour composed of giant spindle and polygonal cells with dark nuclei. By immunohistochemistry (IHC), the tumour cells intensively expressed S-100 protein and vimentin, but lacked melanosome protein HMB45, pan-cytokeratin AEl/AE3 (CK), desmin as well as estrogen and progesterone receptors. Considering the immunophenotype and cellular atypia, malignant peripheral nerve sheath tumour of the breast was diagnosed. There were no subcutaneous nodules, cafe au lait spots, Lisch nodules or history of neurofibromas. Clinically, thyroid mass was also discovered during the diagnostic work-up. The thyroid core biopsy yielded overtly malignant tissue with different structure, composed of atypical small cells. By IHC, these cells expressed CK. Proliferation fraction by Ki-67 (clone MIB-1) was $87 \%$ of the tumour cells. The diagnosis of high-grade thyroid carcinoma was made. Total thyroidectomy was performed as recommended by oncological council. Grossly paradoxically circumscribed high-grade carcinoma, measuring $9 \times 7 \times 5.7 \mathrm{~cm}$ and showing angioinvasion and capsule penetration, was found in the right side of the thyroid. The tumour showed small cell morphology as well as intense expression of CK and synaptophysin, focal expression of chromogranin A, nuclear p53 expression and high proliferation fraction $(84 \%)$ by IHC (Figure 1). No evidence of multifocality was found. Metastases were present in cervical lymph nodes (2/4). The findings were consistent with medullary thyroid carcinoma, small cell variant, pT3N1M0. Right mastectomy with axillary lymph node dissection was performed 10 days after thyroidectomy. Grossly, $11 \times 7 \times 8 \mathrm{~cm}$ firm node was found in the breast, and $9.5 \times 4 \times 6 \mathrm{~cm}$ - in axillary tissues. The tissue structure (Figure 2) was consistent with anaplastic sarcoma. By IHC, intense cytoplasmic and nuclear S-100 expression, nuclear p53 expression as well as low proliferation fraction (5\%) was found. No metastases were found in 26 axillary lymph nodes. The diagnosis of breast MPNST, pT2aNlM0Gl was issued. The postoperative time was uneventful. The patient was discharged on the third postoperative day for out-patient radiation therapy. Nine months after the mastectomy, increased left cervical lymph nodes were found and removed. Metastases of thyroid carcinoma were detected. One month later the patient died of disseminated thyroid cancer.

\section{DISCUSSION}

Malignant peripheral nerve sheath tumour (MPNST), previously designated neurofibrosarcoma, is a rare tumour with an incidence of 1 per 100,000. It constitutes $5-10 \%$ of soft tissue sarcomas. Approximately half of these tumours in adults arise sporadically de novo. The other half occurs in patients suffering from neurofibromatosis, type I (NFl). The mean age at the 
time of MPNST diagnosis is 37 years, but the patients with NF1 are 10 years younger. MPNST originates from a major or minor peripheral nerve sheath. The most common MPNST locations are the trunk $(51 \%)$, the extremities (45\%) and the head and neck in $4 \%$ of patients (1). Less than 10 de novo cases in breast are described (7).

The correct preoperative diagnosis of MPNST depends on biopsy in order to confirm the malignancy and to identify the histogenesis. The microscopic picture can be suggestive of MPNST but is not absolutely diagnostic. The IHC can be diagnostic or can exclude other malignant tumours (6).

The treatment of MPNST is complete surgical removal. There are no reports in the literature on the role of radiotherapy or chemotherapy for the treatment of MPNST of breast (7) but radiotherapy is recommended for high-grade tumours with negative histological resection margins and for intermediate-grade tumours with close or positive histological resection margins. Chemotherapy is recommended as treatment of metastatic disease and it may be helpful in preoperative settings to decrease unresectable primary tumours (4). The patient had high-grade thyroid cancer consistent with the small cell variant of medullary carcinoma. However, there was no evidence of any multiple endocrine neoplasia (MEN) syndrome embarrassing both the final diagnosis and the etiologic considerations. The patient had combination of three rare diseases: multiple sclerosis (the incidence in the world 1.1-4/100 000) (2), primary MPNST of the breast (less than 10 cases described) and medullary carcinoma (0.03-0.5/100 000) (3). The association by chance only would be highly unlikely. However, the detailed history provides no evidence of the most likely syndromes like NF1, or MEN, or any familial cancer syndrome. Therefore we share our evidence with colleagues to demonstrate a possible new oncological syndrome.

Conflict of interest: None

\section{REFERENCES}

1. Anghileri M, Miceli R, Fiore M, Mariani L, Ferrari A, Mussi C, Lozza L, Collini P, Olmi P, Casali PG, Pilotti S, Gronchi A. Malignant peripheral nerve sheath tumors: prognostic factors and survival in a series of patients treated at a single institution // Cancer, 2006; 107: 1065 - 1074

2. Anonymous. Atlas multiple sclerosis resources in the world 2008. Geneva: WHO Press; 2008; 14

3. Chan JKC. Tumors of the thyroid and parathyroid glands // In: Fletcher CDM. Diagnostic Histopathology of Tumors. 3rd ed. Philadelphia: Churchill Livingstone Elsevier; 2007; 997 - 1097

4. Elsaify WM, Elsaify MM, Melek RK. De novo malignant peripheral nerve sheath tumor of the breast: case report number one // European Surgery, 2007; 39: 192 - 195

5. Ferlay J, Autier P, Boniol M, Heanue M, Colombet M, Boyle P. Estimates of the cancer incidence and mortality in Europe in 2006 // Annals of Oncology, 2007; 18: $581-592$

6. Rosai J. Soft tissues // In: Rosai J. Rosai and Ackerman's Surgical Pathology. 9th ed. Edinburgh: Mosby; 2004. p. 2237 - 2371

7. Yi JM, Moon EJ, Oh SJ, Lee A, Suh YJ, Baek JM, Choi SH, Jung SS. Malignant peripheral nerve sheath tumor of the breast in a patient without neurofibromatosis: a case report // Journal of Breast Cancer, 2009; 12: $223-226$

\section{Acknowledgements}

This work was supported by ESF project Nr. 2009/0230/1DP/1.1.1.2.0/09/APIA/VIAA/070.

A.V. was supported by ESF fellowship, project Nr. 2009/0147/1DP/1.1.2.1.2/09/IPIA/VIAA/009.

\section{Address:}

Arnis Abolins

Hereditary Cancer Institute,

Riga Stradins University,

Dzirciema Street 16, LV-1007,

Riga, Latvia

E-mail: arnis.abolinsh@inbox.lv 

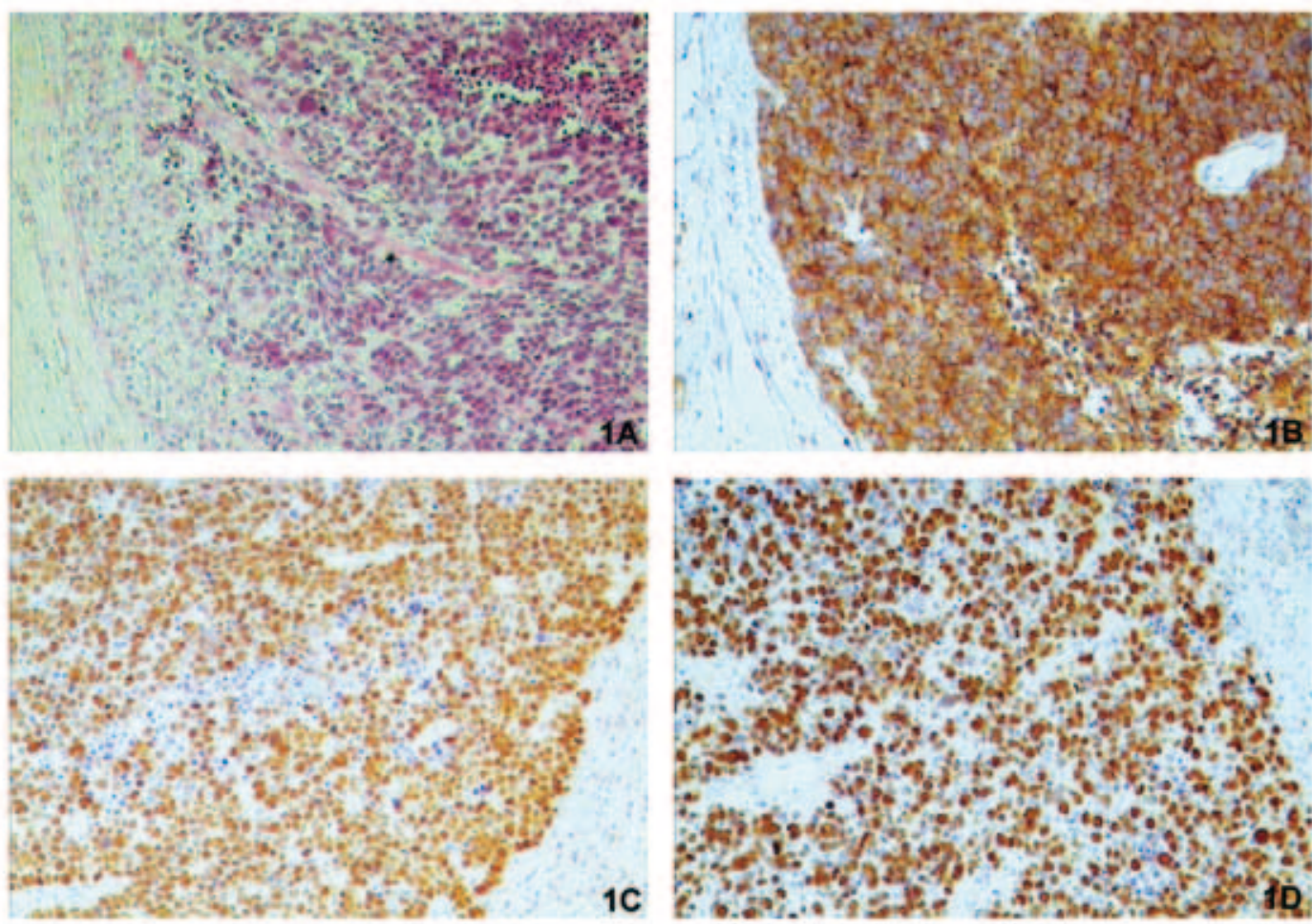

Fig. 1. Medullary thyroid cancer, small cell variant. A, Haematoxylin-eosin, original magnification (OM) x100. B, Synaptophysin, immunoperoxidase (IP), OM X100. C, Aberrant p53, IP, OM x100. D, Proliferation fraction by Ki-67, IP, OM x100
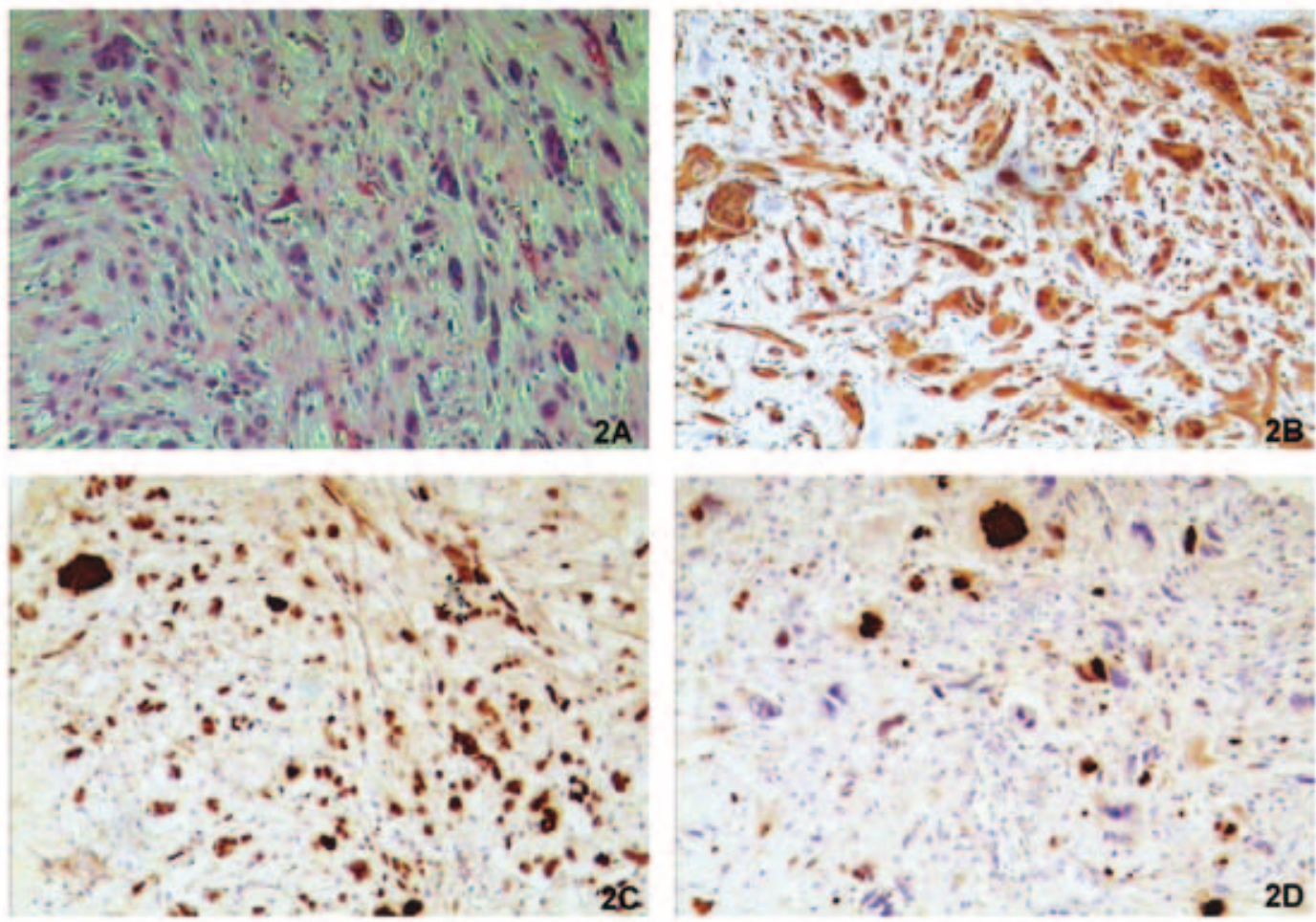

Fig. 2. Malignant peripheral nerve sheath tumour of the breast. A, Haematoxylin-eosin, original magnification (OM) x100. B, S-100 protein, immunoperoxidase (IP), OM X100. C, Aberrant p53, IP, OM x100. D, Proliferation fraction by Ki-67, IP, OM x100 